\title{
Comparative Gain Analysis of Erbium and Ytterbium Doped Optical Fibre Amplifiers
}

\author{
Tanvi \\ M.E. Student \\ PEC University of Technology \\ Sector 12, Chandigarh
}

\author{
Neena Gupta, Ph. D \\ Professor \\ PEC University of Technology \\ Sector 12, Chandigarh
}

\begin{abstract}
In the present work, gain of EDFA (Erbium Doped Fibre Amplifier) and YDFA (Ytterbium Doped Fibre Amplifier) are analyzed based on their performance parameters. The effects of pump laser power, length of doped fibre that used for amplification and the wavelength of the input signal on the gains of the optical amplifiers is examined. EDFA exhibits maximum gain of $48 \mathrm{db}$ around $1550 \mathrm{~nm}$ regions with pump wavelength of $980 \mathrm{~nm}$ (pump power=10Watt, EDF length $=30 \mathrm{~m}$ ), on the other hand YDFA exhibits maximum gain of $62 \mathrm{db}$ around $1030 \mathrm{~nm}$ regions with pump wavelength of $975 \mathrm{~nm}$ (pump power $=5 \mathrm{Watt}$, YDF length $=8 \mathrm{~m}$ ). The ANALYSIS shows that the gain of YDFA is superior to that of EDFA for short fibre length.
\end{abstract}

\section{Keywords}

ytterbium-doped fibre (YDF), erbium-doped fibre (EDF), dense wavelength division multiplexing (DWDM), amplified spontaneous emission (ASE), optical fibre amplifiers (OFA).

\section{INTRODUCTION}

When an optical signal moves along an optical fibre over long distances and if the speed of data is high enough, it gets attenuated along the fibre and gets distorted due to dispersion respectively [1][2]. In order to counterbalance the attenuation losses in the optical fibre, optical fibre amplifiers OFA's came into existence. The development of optical amplifiers started in early 1980s and their use for long-haul communication systems became inescapable during late 1990s. Optical amplifier is a device or a component that amplifies an optical signal directly, without converting into electrical form. An optical amplifier may be assumed as a laser in which feedback from the cavity is suppressed, or a laser without an optical cavity. OFA's must be designed to amplify the signal along the fibre, the more the gain; the more span distance between amplifiers as long as the signal is not distorted due to high optical power. To make use of this great bandwidth, dense wavelength division multiplexing DWDM is used, but each type of optical fibre amplifier has different bandwidth, different range [3][4].

\section{DOPED FIBRE AMPLIFIERS}

The optical fibre can be doped with any rare earth element to provide large gain such as Erbium(Er), ytterbium (Yb), Neodymium(Nd) or Praseodymium(Pr). The host fibre material can be either silica or fluoride based glass. Amplification is achieved by stimulated emission of photons from dopant ions in the doped fibre. The pump laser excites dopant ions present in the doped fiber to a higher energy level from where they decay to a lower energy state (level) by 3 methods. Firstly, the excited ions decay via stimulated emission of a photon at the signal wavelength, which is requirement of amplification. Secondly, the excited ions can also decay by spontaneous emission or thirdly by nonradiative (without light radiations) processes which involves interactions of phonons with the glass matrix which compete with stimulated emission reducing the overall efficiency of amplification of an amplifier. This spontaneous emission amplified along with signal when travels through doped fiber and becomes amplified spontaneous emission (ASE) which is major source of noise. The amplification window or operating regions of an optical amplifier is the range of optical wavelengths for which the amplifier provides a useful gain. The operating regions of an optical amplifier are determined by the spectroscopic properties of the dopant ions, the glass matrix of the optical fibre, power of the pump laser, and the wavelength of pump source [5]. The most popular material for long haul telecommunication applications is a silica fibre doped with Erbium and Ytterbium, which is known as EDFA and YDFA respectively. In Erbium Doped Fibre Amplifier (EDFA), core of a silica fibre is doped with trivalent Erbium ions and can be efficiently pumped with a laser at a wavelength of $980 \mathrm{~nm}$ or $1480 \mathrm{~nm}$, and display gain in the 1500-1640nm regions and in Ytterbium Doped Fibre Amplifier (YDFA) core of a silica fibre is doped with trivalent Ytterbium ions and can be efficiently pumped with a laser at a wavelength of $910 \mathrm{~nm}$ or $975 \mathrm{~nm}$, and display gain in the 1000 $1150 \mathrm{~nm}$ regions.

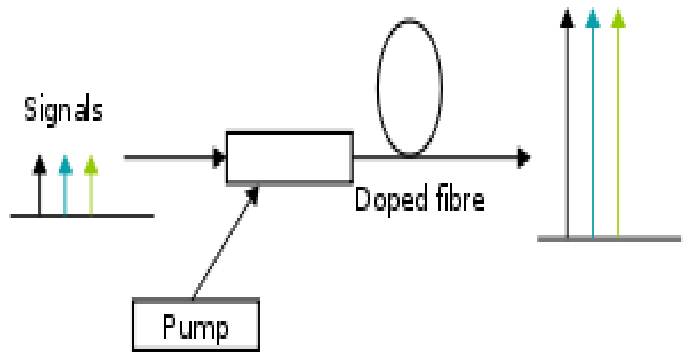

Fig 1: simple doped fibre

\subsection{YDFA System}

Figure 2 Shows the absorption and emission cross-sections of $\mathrm{Yb}^{3+}$ in a silica glass. There are two absorption peaks at $910 \mathrm{~nm}$ and $975 \mathrm{~nm}$ wavelengths and two emission peaks at $975 \mathrm{~nm}$ and $1030 \mathrm{~nm}$ wavelengths. For the superlative pursuance of an amplifier the pump wavelength should be positioned in the range of absorption peak and the 
amplification wavelength should be in the range of emission peaks. Hence by applying pumping wavelength around $910 \mathrm{~nm}$ a very high gain can be achieved at $975 \mathrm{~nm}$ signal wavelength. But, the amplification bandwidth around $975 \mathrm{~nm}$ is narrow which limits the many potential applications. Second possibility is to achieve amplification from 1000 to $1150 \mathrm{~nm}$ signal wavelength (which is broad bandwidth) with a pumping wavelength around $910 \mathrm{~nm}$. Yb ions effectively excited by $910 \mathrm{~nm}$ pumping, providing inversion close to $97 \%$ for high pump powers. As a result very high gains can be achieved in very short fibre length. However, the gain spectrum for $910 \mathrm{~nm}$ pumping contains a high peak at $975 \mathrm{~nm}$, which give rise to strong amplified spontaneous emission (ASE) around $975 \mathrm{~nm}$ which is major source of noise. This ASE diminishes the inversion and reduces the gain as the fibre length increases, which could reduce the overall proficiency significantly. The advantage of using $975 \mathrm{~nm}$ pumping wavelength is that it does not contain the $975 \mathrm{~nm}$ ASE peak, because the absorption and emission cross-sections at $975 \mathrm{~nm}$ are nearly the same. The $975 \mathrm{~nm}$ pumps excite $\mathrm{Yb}$ ions by providing inversion close to $50 \%$ which leads to smaller gains for short fibres as compared with $910 \mathrm{~nm}$ pumping. However, the $975 \mathrm{~nm}$ pumped YDF have no problem of $975 \mathrm{~nm}$ ASE which is a major problem, it means that the maximum gain is limited only by ASE around $1030 \mathrm{~nm}$. Hence as the fibre length is increased higher gains can be achieved [6].

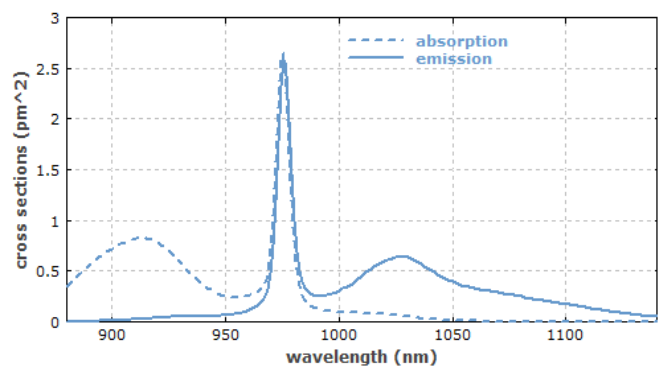

Fig 2: Absorption and emission cross-sections of $\mathbf{Y b}^{3+}$

\subsection{EDFA System}

EDFAs have two commonly-used pumping bands - 980nm and $1480 \mathrm{~nm}$. Absorption cross-section at $980 \mathrm{~nm}$ band is higher and is generally used where low-noise performance is required. As the absorption band is relatively narrow hence wavelength stabilized laser sources are needed. But absorption cross-section for the $1480 \mathrm{~nm}$ band is lower and broader so it is generally used for higher power amplifiers. A combination of $980 \mathrm{~nm}$ and $1480 \mathrm{~nm}$ pumping is generally best blend in amplifiers. EDFA provide high gain in $1530-1560 \mathrm{~nm}$ wavelength band region, here erbium ions emitted band energy is greater than the energy absorbed and in this range gain bandwidth is narrower. The emission and absorption of the energy EDF according to Figure 3 of the spectrum, where the highest gain peak of EDFA near 1550nm, hence the gain obtained clears that the operating wavelength is $1550 \mathrm{~nm}$ [7][8][9].

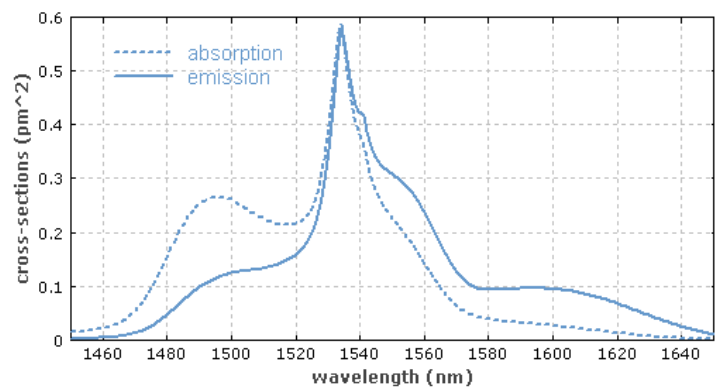

Fig 3: Absorption and emission cross-sections of $\mathbf{E r}^{3+}$

\section{ANALYSIS OF EDFA}

In this section, VPI simulation program is used to prepare model for Figure 4(a) and figure 5(a). For EDFA assembly single stage WDM signal source ranges from (1500-1640nm) with constant signal power, pump source of $980 \mathrm{~nm}$ with different pump power $(1 \mathrm{~W}, 10 \mathrm{~W}$ etc.), erbium doped fibre of variable length $(8 \mathrm{~m}, 10 \mathrm{~m}, 20 \mathrm{~m}, 30 \mathrm{~m}, 50 \mathrm{~m}$ etc. $)$ is considered. Test set amplifier and optical spectrum analyzer are used to measure output power, gain and optical spectrum. In EDFA system (working with band $1500 \mathrm{~nm}$ and $1640 \mathrm{~nm}$ ) when the analysis is done according to EDFA assembly as shown in Figure 4(a) gain start increasing till $1550 \mathrm{~nm}$ and then flattened and after that near $1550 \mathrm{~nm}$ gain rapidly begins to fall as shown in figure 4(b). In Table1: max value of gain for different length of EDF and different pump power is given.

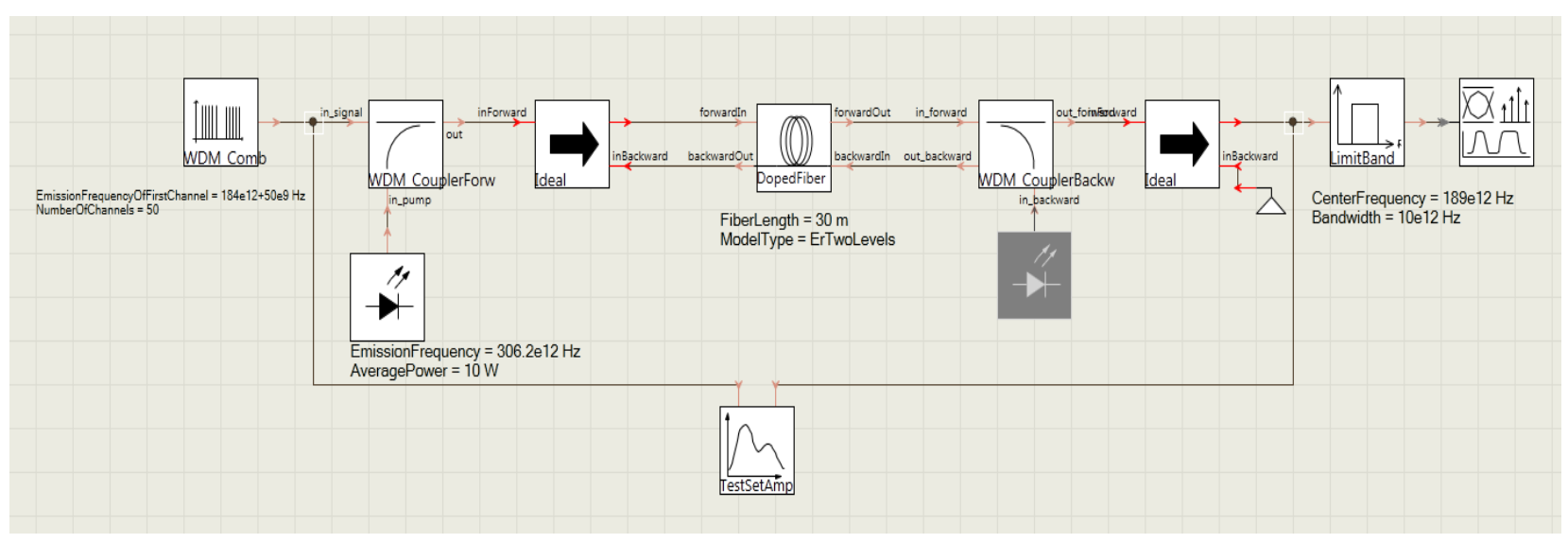

Fig 4(a): EDFA assembly 
Table1: Analysis of EDF gain with single stage

\begin{tabular}{|c|c|c|c|}
\hline Signal Wavelength(nm) & Gain $(\mathbf{d b})$ & Length of Doped Fibre (m) & Pump Power (Watt) \\
\hline 1550 & 33 & 50 & 10 \\
\hline 1550 & 28 & 8 & 10 \\
\hline 1550 & 41 & 20 & 1 \\
\hline $\mathbf{1 5 5 0}$ (Best Case) & $\mathbf{4 8}$ & $\mathbf{3 0}$ & $\mathbf{1 0}$ \\
\hline
\end{tabular}

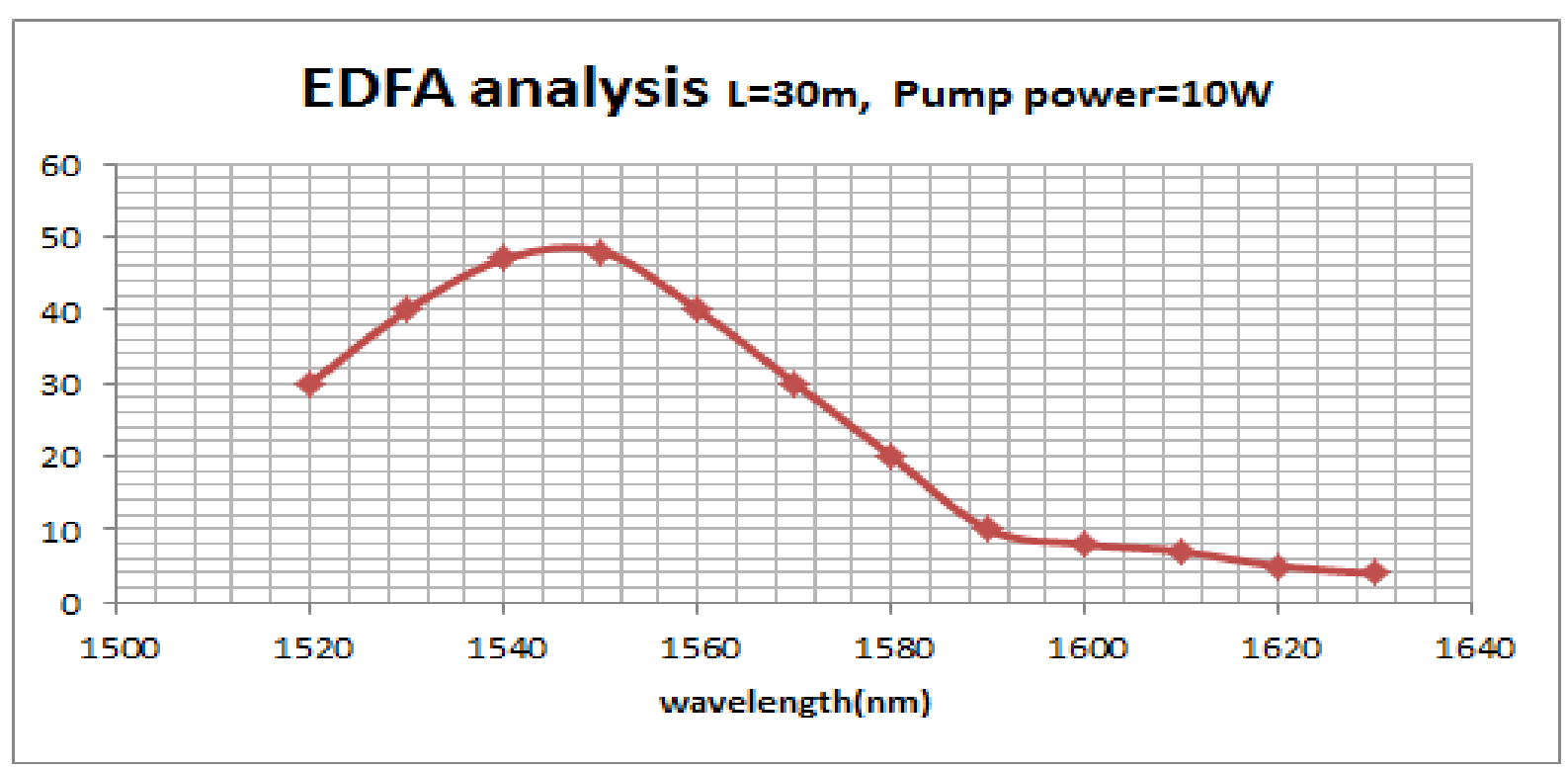

Fig 4(b): Variation in gain (db) of EDFA.

\section{ANALYSIS OF YDFA}

For YDFA assembly single stage WDM signal source ranges from $1000 \mathrm{~nm}$ to $1140 \mathrm{~nm}$ with constant signal power, pump source of $975 \mathrm{~nm}$ with different pump power (1W,5W etc.), Ytterbium doped fibre of variable length $(6 \mathrm{~m}, 8 \mathrm{~m}, 10 \mathrm{~m}$ etc. $)$ is considered. Test set amplifier and optical spectrum analyzer are used to measure gain, output power and optical spectrum.
In YDFA system (working with band $1000 \mathrm{~nm}$ and $1140 \mathrm{~nm}$ ) when the analysis is done according to YDFA assembly as shown in Fig 5(a) gain start increasing firstly till 1030nm and then after gain rapidly begins to fall quickly as shown in figure 5(b). In Table2: max value of gain for different length of YDF, and different pump power is given.

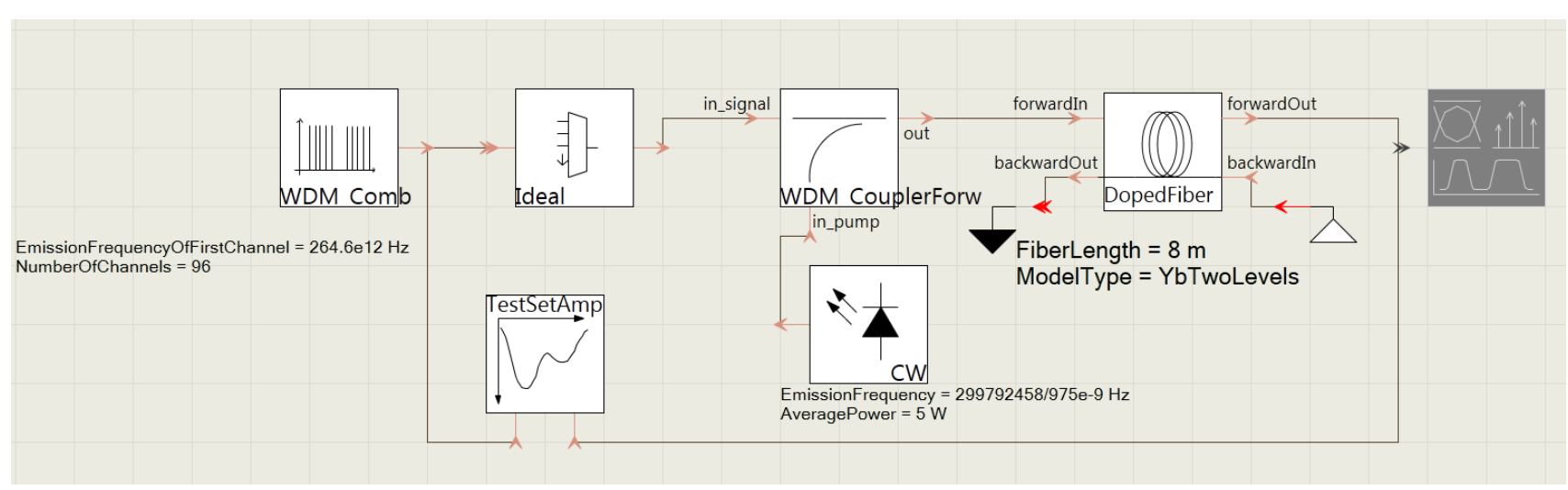

Fig 5(a): YDFA assembly 
Table2: Analysis of YDF gain with single stage

\begin{tabular}{|l|c|c|c|}
\hline Signal Wavelength(nm) & Gain $(\mathbf{d b})$ & Length of Doped Fibre (m) & Pump Power (Watt) \\
\hline 1030 & 52 & 8 & 1 \\
\hline 1030 & 50 & 10 & 1 \\
\hline 1030 & 55 & 6 & 5 \\
\hline 1030 (Best Case) & $\mathbf{6 2}$ & $\mathbf{8}$ & $\mathbf{5}$ \\
\hline
\end{tabular}

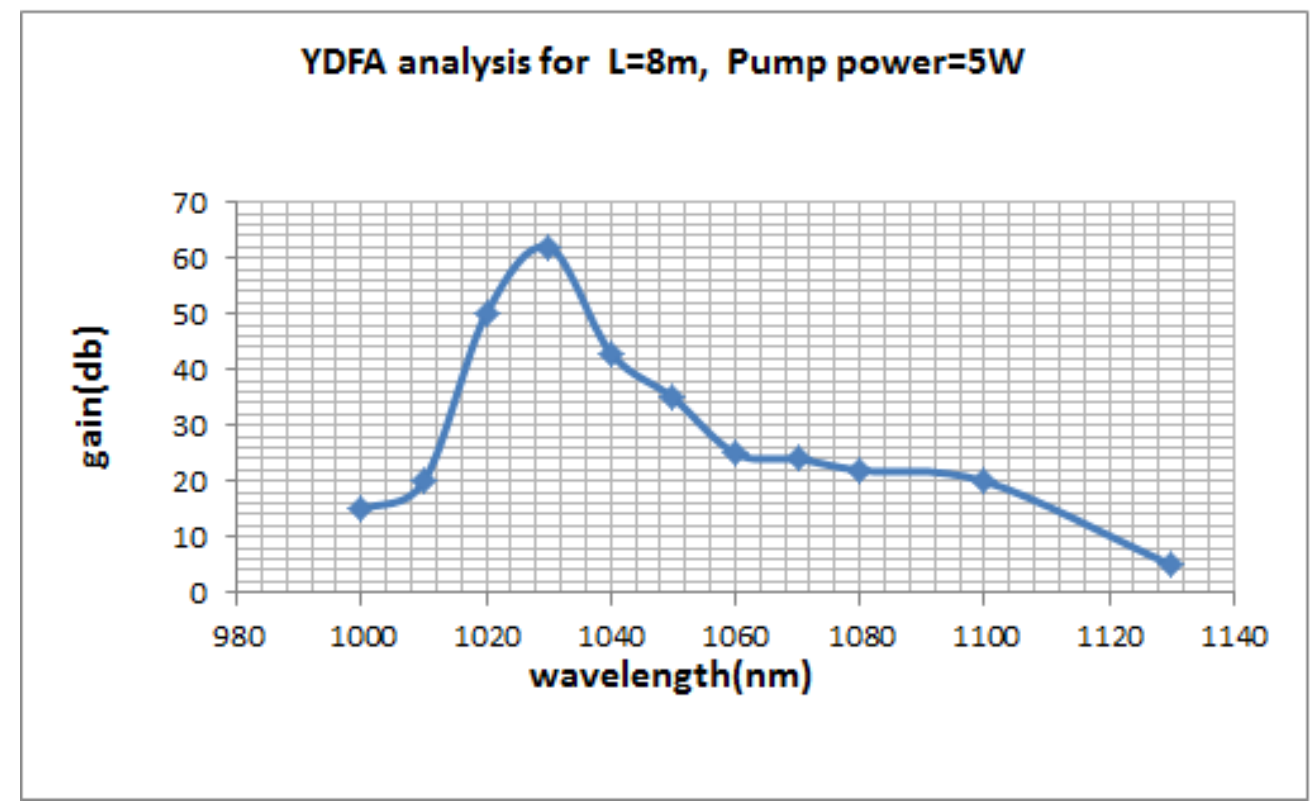

Fig 5(b): Variation in gain (db) of YDFA.

\section{CONCLUSION}

Optical amplifiers are very important module in long distance optical communication. In this paper gain characters of EDFA (Erbium Doped Fibre Amplifier) and YDFA (Ytterbium Doped Fibre Amplifier) examined with constant/fixed signal power. EDFA maximum gain $48 \mathrm{db}$ with $30 \mathrm{~m}$ length of fibre around $1550 \mathrm{~nm}$ signal wavelength and pump power of 10 watt while in YDFA $62 \mathrm{db}$ gain is achieved with only $8 \mathrm{~m}$ fibre length and pump power of 5 watt around $1030 \mathrm{~nm}$ signal wavelength. Hence YDFA is better than EDFA. Length of doped fibre required in case of YDFA is less as compared to EDFA. It is due to the reason that Ytterbium doped fibre has two main energy levels involved in the light amplification and gain media arise from the fact that only one excited state is involved in the laser transition [10]. The comparatively small energy gap between the lower energy level and excited-state results in extremely low quantum defects, consequently high power efficiency is possible, and many detrimental effects such as thermal effects, quenching and excited state absorption are meaningfully condensed [11]. But Erbiumdoped fibre has three main energy levels involved in the light amplification [12] and in EDFA excited state absorption and concentration quenching phenomenon is present at large extent, So YDFA can emit high output power only using a small fibre lengths.

\section{FUTURE SCOPE}

In this paper only single pass assembly of EDFA and YDFA is considered but in future double pass, triple pass and higher configuration can be examined which may exhibits large gain around 90db. Moreover we can see gain of YDFA and EDFA is not flat. To flatten the gain many techniques can be implemented and gain equalizing filters can be used. To increase the input signal range many amplifiers like RAMAN+YDFA, EDFA+RAMAN, RAMAN+YDFA+RAMAN, SOA+YDFA etc. hybrid connection can be considered, which will provide not only longer range amplifier but may also give increased and flattened gain.

\section{REFERENCES}

[1] Gerd Keiser, "optical fibre Communications", 4th Edition, Tata McGraw-Hill Education Pvt. Ltd., New Delhi, Inc. 2009, ISBN-13: 978-0-07064810-4.

[2] Govind P. Agarwal, "Fibre Optic Communication Systems", John Wiley \& sons, Inc. Publication, 2003.

[3] D.K.Mynbaev, et.al, "Fibre Optics Communications Technology", Pearson Education, Delhi, Inc. 2003, ISBN 81-7808-317-5. 
[4] John N. Senior, "Optical Fibre Communications principles and practice", New Delhi, 2005.

[5] Sunil Kumar Panjeta, et.al, "Gain Optimization of EDF Optical Amplifier by Stages Enhancement and Variation in Input Pumping Power", International Journal of Scientific and Research Publications, ISSN 2250-3153, vol. 2, no. 11, November 2012.

[6] Paschotta, R., et.al, "Ytterbium-doped fibre amplifiers", IEEE Journal of Quantum Electronics, vol. 33, no. 7, pp. 1049-1056, 1997.

[7] Naji W.A, et.al, "Review of Erbium-doped fibre amplifier", International Journal of Physical Sciences Vol. 6, no. 20, pp. 4674-4689, September 23, 2011.

[8] S.A.Daud, et.al, “An Experimental Study on S-band Depressed Cladding EDFA", Proceedings of IEEE 2008 $6^{\text {th }}$ National Conference on Telecommunication
Technologies and IEEE $20082^{\text {nd }}$ Malaysia Conference on Photonics, 26-27 August 2008, Putrajaya, Malaysia.

[9] Semmalar S., Poonkuzhali, Devi.P, “Optimized Gain EDFA of different lengths with an influence of pump power", IEEE Paper, 2011 Novak.

[10] H.M.Pask, et.al, "Ytterbium-doped silica fibre lasers :versatile sources for the $1-1.2 \mu \mathrm{m}$ region", IEEE Journal Selected Topics in Quantum Electronics, vol.1, no.1, pp.2-13, 1995.

[11] R.Paschotta, "Encyclopedia of laser physics and technology."

[12] http://www. rp-photonics.com/encyclopedia.html

[13] Y.Sun, J.L.Zyskind and A.K.Srivastava, "Average Inversion Level Modeling and Physics of Erbium-Doped Fibre Amplifiers", IEEE Journal of Selected Topics in Quantum Electronics, vol.3, no.4, August 1997. 